Short communication

\title{
Cellular fate of deformable needle-shaped PLGA-PEG fibers
}

\author{
Bokai Zhang ${ }^{\mathrm{a}, \mathrm{c}}$, Mingliu Zhu ${ }^{\mathrm{b}, \mathrm{g}}$, Zhi Li ${ }^{\mathrm{c}, \mathrm{f}}$, Ping Sai Lung ${ }^{\mathrm{a}}$, Wojciech Chrzanowski ${ }^{\mathrm{d}}$, \\ Chi Tat Kwok ${ }^{\mathrm{e}}$, Jian $\mathrm{Lu}^{\mathrm{b}, \mathrm{h}, *}$, Quan $\mathrm{Li}^{\mathrm{a}, *}$ \\ a Department of Physics, The Chinese University of Hong Kong \\ ${ }^{\mathrm{b}}$ Department of Mechanical Engineering, City University of Hong Kong, Kowloon, Hong Kong, China \\ 'Shenzhen Key Laboratory of Nanobiomechanics, Shenzhen Institutes of Advanced Technology, Chinese Academy of Sciences, China \\ d Faculty of Pharmacy, The University of Sydney \\ ${ }^{\mathrm{e}}$ Department of Electromechanical Engineering, University of Macau \\ f State Key Laboratory of Mechanics and Control of Mechanical Structures, Nanjing University of Aeronautics E Astronautics, Nanjing, 210016, Jiangsu, China \\ ${ }^{g}$ State Key Laboratory of nonlinear mechanics, Institute of Mechanics, Chinese Academy of Sciences, No.15, West Road, North $4^{\text {th }}$ Ring, Beijing, 100190, China

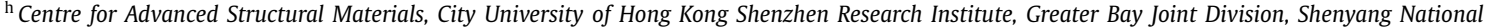 \\ Laboratory for Materials Science, Shenzhen, 518057, China
}

\section{A R T I C L E I N F O}

\section{Article history:}

Received 7 January 2020

Revised 15 May 2020

Accepted 19 May 2020

Available online 26 May 2020

\section{Keywords:}

Deformability

Apparent Young's modulus

Actin revolution

\begin{abstract}
A B S T R A C T
Deformability of micro/nanometer sized particles plays an important role in particle-cell interactions and thus becomes a key parameter in carrier design in biomedicine application such as drug delivery and vaccinology. Yet the influence of material's deformability on the cellular fate of the particles as well as physiology response of live cells are to be understood. Here we show the cellular fate of needle shaped (high aspect ratio 25) PLGA-PEG copolymer fibers depending on their deformability. We found that all the fibers entered murine macrophage cells (RAW 264.7) via phagocytosis. While the fibers of high apparent Young's modulus (average value $=872 \mathrm{kPa}$ ) maintained their original shape upon phagocytosis, their counterparts of low apparent Young's modulus (average value $=56 \mathrm{kPa}$ ) curled in cells. The observed deformation of fibers of low apparent Young's modulus in cells coincided with abnormal intracellular actin translocation and absence of lysosome/phagosome fusion in macrophages, suggesting the important role of material mechanical properties and mechano-related cellular pathway in affecting cell physiology.
\end{abstract}

\section{Statement of Significance}

Particles are increasingly important in the field of biomedicine, especially when they are serving as drug carriers. Physical cues, such as mechanical properties, were shown to provide insight into their stability and influence on physiology inside the cell. In the current study, we managed to fabricate 5 types of needle shaped PLGA-PEG fibers with controlled Young's modulus. We found that hard fibers maintained their original shape upon phagocytosis, while soft fibers were curled by actin compressive force inside the cell, causing abnormal actin translocation and impediment of lysosome/phagosome fusion, suggesting the important role of material mechanical properties and mechano-related cellular pathway in affecting cell physiology.

(C) 2020 Acta Materialia Inc. Published by Elsevier Ltd. All rights reserved.

\section{Introduction}

Physical attributes of particles have recently emerged as important parameters in determining their cytotoxicity as well as designing them for various bio-applications such as drug delivery

\footnotetext{
* Corresponding author.

E-mail address: jianlu@cityu.edu.hk (J. Lu).
}

and bio-sensing. These attributes, including the size [1-3], surface charge [4] and chemistry [5,6], shape [7-12], and mechanical properties [13], not only determine the cellular fate of particles, but also affect the physiology of the cells [14]. Among them, the size, shape and surface charge/chemistry effect have been well documented in the literature [11,15-17] since advances in particle synthesis [18] have facilitated systematic studies that investigate their influence as being drug carry. Beyond these researches, 
less progress have been made on how mechanical properties of the particles affect their interaction with the cells, even though mechanical property of bulk material in other field is well understood, especially in tissue engineering [19].

In fact, cells have been known to have mechano-sensors and pathways for specific physiological processes $[20,21]$. One representative example is the anchoring of particles to the cell membrane to trigger cellular uptake. It has was reported that the stiffer particles with compressive modulus of $155.7 \mathrm{kPa}$ resulted in stronger obstacle on the cell adhesion ability than the softer particles of $16.7 \mathrm{kPa}$ [22]. After anchoring, the cellular uptake was reported to also depend on the particles' mechanical properties. One of the most obvious express of particles' mechanical properties is their deformation, whether particles undergo deformation provides insight into their stability and the potential mechanism of action inside the cell [23], especially when serving as drug carriers. Softer particles are more likely to deform under pressure during the uptake process [24-26]. For example, preferable internalization in macrophage cells were found by using nanoparticles with Young's modulus of $3000 \mathrm{kPa}$ vs. those of $10 \mathrm{kPa}$, as the later ones tend to deform in such a way that the particle's radius of curvature changes in the direction normal to the cell membrane, avoiding uptake by macrophage [27]. This feature associated with the softer nanoparticles lead to their longer circulation half-life in blood stream [27,28] to achieve "stealth" to macrophage. The more difficult cellular uptake of softer nanoparticles is understood by the larger adhesion energies needed to complete the membrane wrapping during endocytosis in simulation models $[29,30]$. However, opposite conclusion has been drawn from a recent work of pickering emulsion droplets with force-dependent deformation property, when enhanced cellular uptake and thus immune response was identified due to the droplets were deformed by the cellular wrapping to enlarge the contact zone with cellular membranes that triggered three-dimensional multivalent interactions with antigen-presenting cells, and subsequently stimulated potent innate and adaptive immune responses [31]. All of the scattered results aroused the importance of understanding particles' mechanical properties effect on cells for bioapplications.

In this study, the cellular fate of needle shaped Poly (lacticco-glycolic acid, LA:GA=1:1) polyethylene glycol (PLGA-PEG) fibers and the physiological responses of the cells were investigated by studying their interaction with the murine macrophage RAW 264.7 cells. In traditional studies, the used spherical shaped particles suffered from the difficulty in observation of deformation in optical means. Thus needle shaped fibers in this study was another solution due to their length is larger than the optical resolution. Apparent Young's modulus is a physical parameter commonly employed to describe the deformability of materials in the elastic regime. Here we manipulated the apparent Yong's modulus by varying the PLGA molecule chain length in the composite, then applied nano-precipitation/solvent diffusion method and stretching method to turn the PLGA-PEG polymers to fiber shape. We used AFM to measured their apparent Young's modulus and confirmed their mechanically inhomogeneous. After incubating with cells, we observed distinct features of the intercellular trafficking of the fibers. Consistently, the cells showed different physiological responses upon the fibers' internalization. In particular, we observed deformation of the softer (average value $=56$ \& $142 \mathrm{kPa}$ ) PLGA-PEG fibers when being trapped inside actinbounded vesicles, which was absent in the case of harder (average value $=246,434 \& 872 \mathrm{kPa}$ ) fibers. This provided us with a possible in situ method to evaluate the mechanical property of actin coatings in live cells. On the other hand, the deformation of the fibers triggered by actin activity suggested a possible parameter in carrier design for stimuli-responsive intracellular cargo release.

\section{Experimental methods}

Preparation and characterization of needle shaped PLGA-PEG fibers

PLGA-PEG copolymer was firstly synthesized into spherical shape using the nano-precipitation/solvent diffusion method [32], the spherical shaped particles were then stretched into needle shape using the stretching methods [8]. Refer to SI for more information.

Scanning electron microscopy (SEM, FEI Quanta 400) was used to obtain the morphology of the needle shaped PLGA-PEG fibers with the accelerating voltage of $2 \mathrm{kV}$. Atomic force microscopy (AFM, Bruker BioScope Catalyst) was applied to measure both the morphology and apparent Young's modulus via Peak Force QNM mode at $37{ }^{\circ} \mathrm{C}$ in PBS simultaneously. Fourier-transform infrared spectroscopy (FTIR, Thermo Nicolet 6700) was used to character. Dynamic light scattering (DLS, Beckman coulter, Delsa ${ }^{\mathrm{TM}}$ Nano) was used to measure the surface charge and mobility. Gel permeation chromatography (K-Alpha, Thermo Scientific) was used to measure the molecular weight. Photoluminescence (Hitachi P7000) was applied to verify the conjugation of Nile red inside the fibers, and to detect the fluorescence intensity of each kind of fibers at the same concentration. Refer to SI for more information.

\section{Introducing PLGA-PEG fibers to cells}

RAW 264.7 (ATCC ${ }^{\circledR}$ TIB-71 ${ }^{\mathrm{TM}}$ ) murine macrophage cells were used in this study. The cells were cultured with Dulbecco's modified Eagle's media (Life technology), supplemented with10 \% fetal bovine serum (Life technology). Cells were grown in a standard cell culture incubator at $37^{\circ} \mathrm{C}$ with $5 \% \mathrm{CO}_{2}$ in a humidified atmosphere. Cells were allowed to be incubated for 24 hrs before the needle shaped PLGA-PEG fibers were introduced. All the fibers were sterilized using UV light for 15 min before feeding. The feeding concentration of the fibers was always kept at $100 \mu \mathrm{g} / \mathrm{mL}$ unless otherwise specified.

\section{Cellular uptake analysis}

The concentration dependent cellular uptake of needle shaped PLGA-PEG fibers was carried out by feeding RAW 264.7 cells with the fibers at different concentrations $(2.5,5,10,25,50$ and 100 $\mu \mathrm{g} / \mathrm{mL}$ ) for 3 hrs, cells were then washed twice with PBS and analyzed by flow cytometry with excitation of $488 \mathrm{~nm}$. The time dependent cellular uptake of needle shaped PLGA-PEG fibers was measured by flow cytometry after the cells being fed with the fibers for different time intervals (i.e., $0.16,0.5,1,3,6,10$ and 14 hrs).

To determine the cellular uptake mechanism of the needle shaped PLGA-PEG fibers, RAW 264.7 cells were firstly incubated in serum free medium in the presence of special inhibitors, including sodium azide, chlorpromazine, genistein, amiloride and cytochalasin D, whose concentration is given in Table S1 for $3 \mathrm{hrs}$, and then co-incubated with the fibers for another 6 hrs, cells were finally washed twice with PBS, and analyzed by flow cytometry.

\section{Physiological change}

All biochemical assays were started from seeding the cells in 96 well plate at the concentration of 5000 cells pre well for 24 hrs, then the needle shaped PLGA-PEG fibers were added for another $14 \mathrm{hrs}$ incubation. Cell membrane perturbation was studied by LDH release assay (CytoTox 96 Non-Radioactive Cytotoxicity Assay, Promega), the cytotoxicity caused by the fibers was evaluated using MTT assay. 


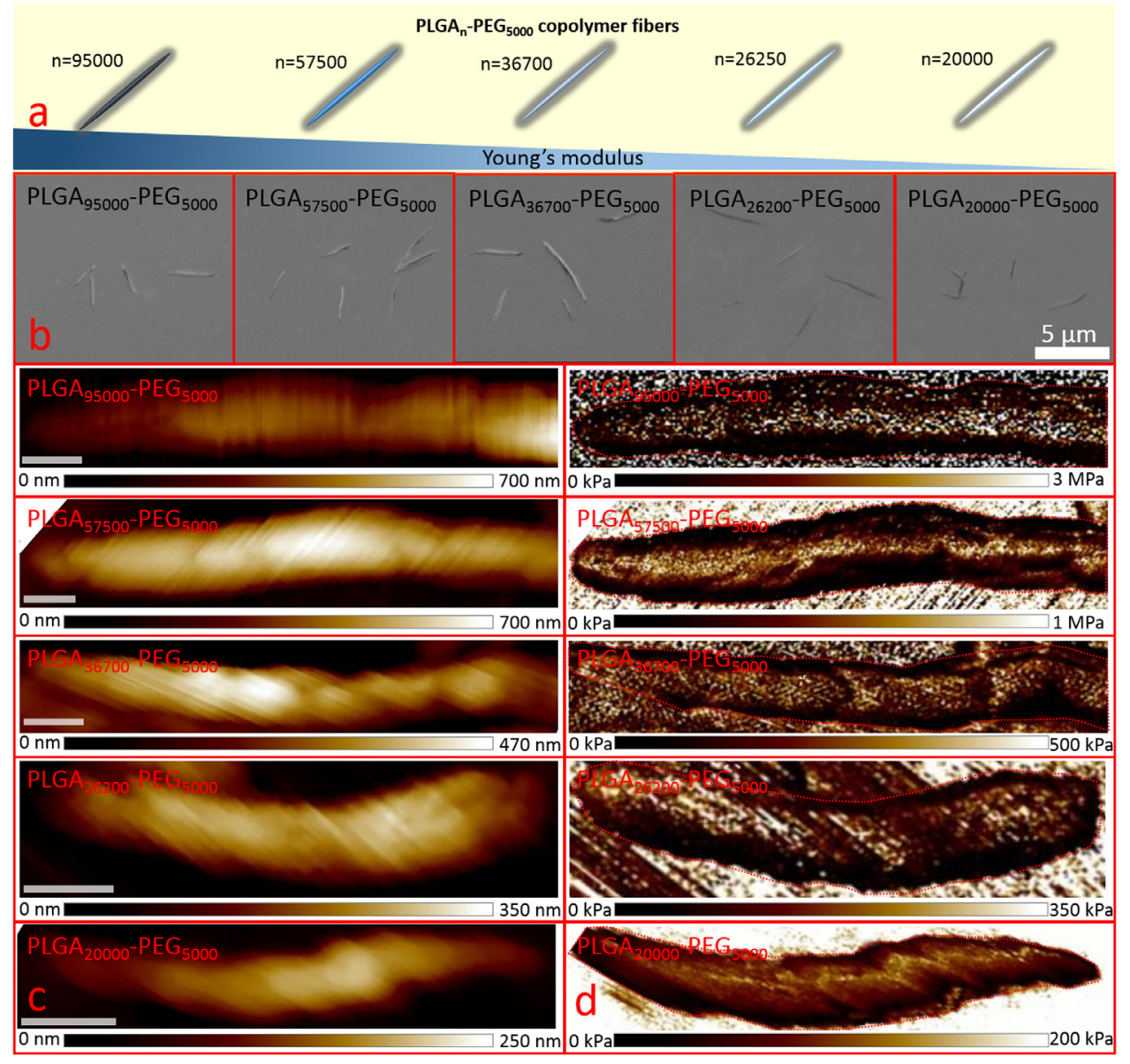

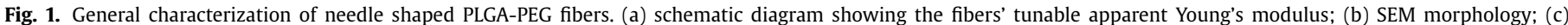
AFM height images; and (d) the corresponding Young's modulus mapping. (both (c) and (d) share the same scale bar: 500 nm).

Confocal laser scanning microscopy (CLSM, Leica SP5TCS II) was used to study the time dependent cellular uptake and intracellular distribution of the fibers, and the revolution of actin affected by the fibers. For the former one, cells were stained with Dextran-FITC (Invitrogen) $1 \mathrm{hr}$ before the feeding of the fibers, then the cells were collected at different time intervals, fixed with $4 \%$ formaldehyde, and observed by CLSM (Ex $488 \mathrm{~nm}$, Em: $516 \mathrm{~nm}$ for DextranFITC, $650 \mathrm{~nm}$ for fibers); for the latter one, the fibers fed cells were collected at different time interval, fixed with $4 \%$ formaldehyde, permeabilized with $0.1 \%$ Triton X-100,and stained with Phalloidin (Invitrogen), then observed by CLSM (Ex 488nm, Em: $516 \mathrm{~nm}$ for Phalloidin, 650 for fibers).

\section{Simulation of actin compressive force}

The ABAQUS, finite element analysis (FEA) software, was employed to simulate the buckling of the PLGA-PEG fiber. The diameter and length of the fiber were set to $200 \mathrm{~nm}$ and $5 \mu \mathrm{m}$ with ununiformed mechanic distribution, considering the indentation of the AFM measurement was set to $20 \mathrm{~nm}$, the resulted Young's modulus represented the surface mechanics. To simplify the model, we assume the inner core to be harder and with diameter of 180 $\mathrm{nm}$, the outer layer to be softer and ununiformed, with two $10 \mathrm{~nm}$ thickness layers. The fiber was divided into five sections along axial direction evenly. Every section of the core was the same in geometry but different in elasticity modulus that varying from 100 to $140 \mathrm{kPa}$. The outer layers were softer than the core and their elasticity modulus were vary from 5 to $30 \mathrm{kPa}$. 5\% compressive strain was implemented on the fiber. The fiber buckled due to the ununiformed mechanic distribution (Fig. S13).

\section{Statistical analysis}

All data are provided as the mean \pm standard deviation (SD). For the fibers' Young's modulus calculation, Nanoscope software was used to select the fibers in each frame, then Young's modulus of each pixel of the fibers were calculated. Their statistical analysis was performed using the column statistics function in GraphPad Prism, with confidence interval of $95 \%$ to obtain Mean value as average, Std. Error of Mean as the error, and the minimum and maximum number. For flow cytometry analysis, a minimum of 10000 cells per sample was considered. Their statistical analysis was performed using the column statistics function in GraphPad Prism, with confidence interval of $95 \%$ to obtain Mean value as average, $\mathrm{SD}$ as the error.

\section{Results}

General characterizations of PLGA-PEG fibers

The molecular weight of PEG was fixed at $5000 \mathrm{Da}$, while that of PLGA varied from 95000 to 20000 Da to manipulate the apparent Young's modulus, and eventually the deformability of the fibers (Fig. 1a). SEM images were taken from the needle shaped PLGAPEG fibers (Fig. 1b), revealing their similar morphologies despite of the different PLGA molecular weight in the respective fibers. The average diameter of the fibers was $\sim 200 \mathrm{~nm}$, with an aspect ratio 


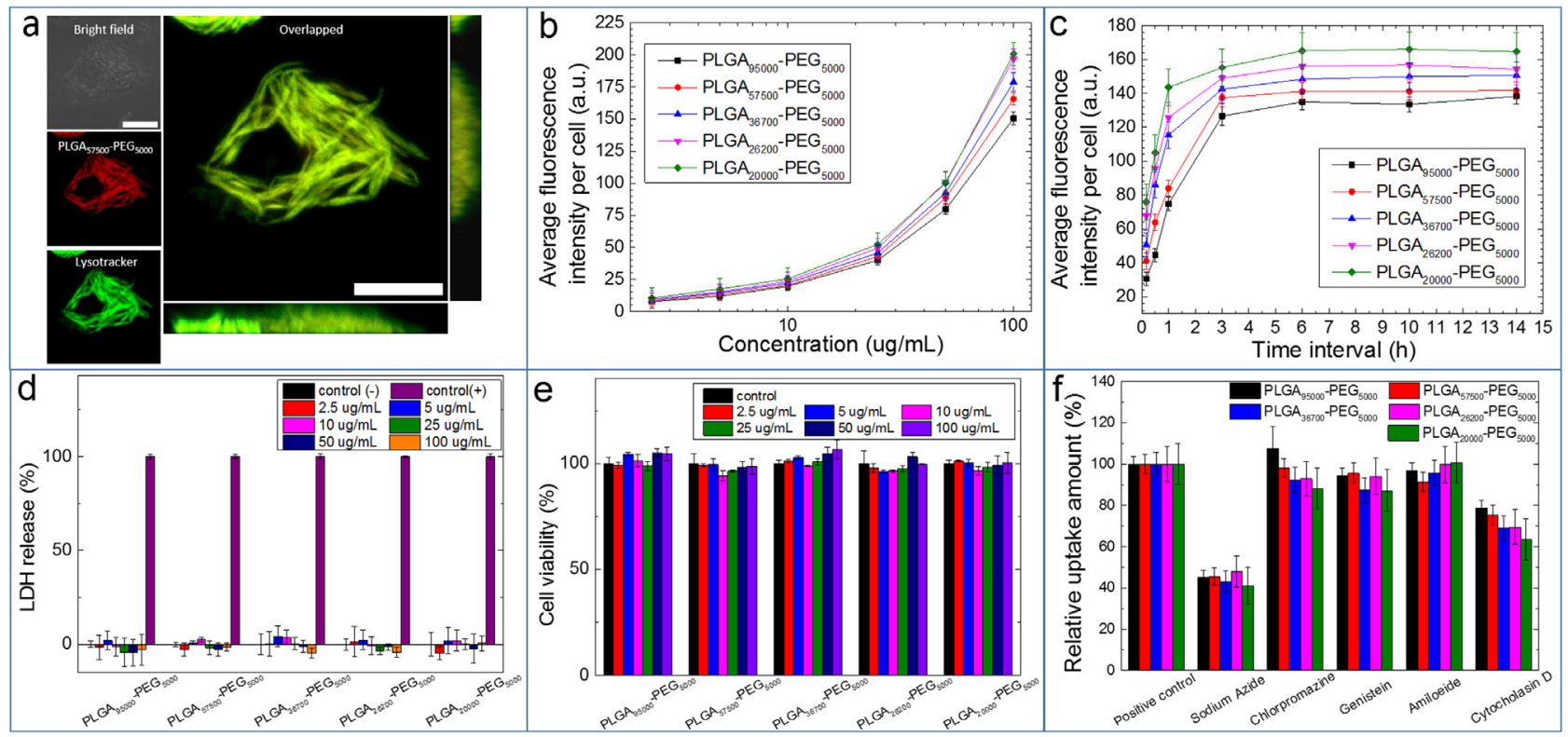

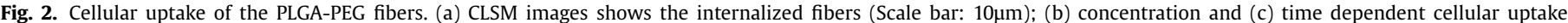
amount of the fibers; (d) LDH release and (e) MTT assay; (f) relative cellular uptake amount with inhibitors.

of $\sim 25$. The tip size of the fiber is described using radius of curvature, which is estimated as $150-250 \mathrm{~nm}$ in PBS from the respective AFM images (Fig. 1c).

Mechanical properties of individual needle shaped PLGA-PEG fibers were measured using AFM. As shown in Fig. 1d, the force mapping of any single fiber revealed the non-uniform local apparent Young's modulus. Taken the hardest and softest fiber as an example, the former one (Fig. S1a) had a broad distribution of measured apparent Young's modulus from 200 to $3000 \mathrm{kPa}$; as a comparison, the latter one (Fig. S1b) showed apparent Young's modulus from $\sim 0$ to $300 \mathrm{kPa}$, but with majority of the fibers having Modulus $\leq 60 \mathrm{kPa}$. The average apparent Young's modules of samples from hard to soft were measured to be $872,434,246,142$ and $56 \mathrm{kPa}$, which represented the average apparent Young's modulus associated with the overall apparent Young's modulus values from Gaussian distribution of each PLGA-PEG fibers population (Table S2).

Surface chemistry of the needle shaped PLGA-PEG fibers was studied by FTIR (Fig. S2a). Signature of both PLGA and PEG were identified in all samples. Quantitative surface analysis of the fibers was done by XPS (Fig. S2b). From the fingerprint in the $C 1 \mathrm{~s} \mathrm{spec-}$ tra, similar $\mathrm{C}-\mathrm{C} / \mathrm{C}-\mathrm{H}, \mathrm{C}-\mathrm{O}$ and $\mathrm{C}=\mathrm{O}$ contents were observed in all the five types of fibers. Stable physicochemical properties of the fibers during the experimental period were confirmed by zeta potential, mobility, molecular weight, average length, aspect ratio and average Young's modulus measured before or after immersion in pH5 citric acid for 14 hrs, as being summarized in Table S2.

Cellular uptake and intracellular deformation of the needle shaped PLGA-PEG fibers

Direct evidence of cellular uptake came from the CLSM studies. After feeding the cells with different fibers for 14 hrs, cells were stained with Lysotracker as the acidic compartment marker, and observed. Using PLGA ${ }_{57500}-\mathrm{PEG}_{5000}$ as an example (Fig. 2a), the yellow color in the CLSM images confirmed the internalized fibers, as this color was from the overlapping of green (florescence of Lysotracker) and red (florescence of fibers) colors. Similar observation was made in all other samples (Fig. S3).

Quantitative results of the cellular uptake amount were obtained by flow cytometry, showing similar trend in all samples, that is, at fixed feeding time (i.e., 3 hrs.), the cellular uptake amount of the needle shaped PLGA-PEG fibers increased gradually with concentration increasing from 2.5 to $25 \mu \mathrm{g} / \mathrm{ml}$. when the concentration was further increased to 50 and $100 \mu \mathrm{g} / \mathrm{mL}$, a surge in the uptake amount took place. Similar cellular uptake behavior was observed for all five samples, although the softer fibers had slightly higher uptake amount at the same concentration when compared to the harder ones, and this only became obvious at high feeding concentrations ( $>25 \mu \mathrm{g} / \mathrm{ml}$ ). (Fig. 2b). At fixed concentration (i.e., $100 \mu \mathrm{g} / \mathrm{mL}$ ), the cellular uptake increased largely for all samples during the first 3 hrs incubation. After that a plateau was reached in all plots. Again, higher uptake amount was observed in the case of softer fibers. (Fig. 2c).

Cytotoxicity of the fibers were studied by both LDH (Fig. 1d) and MTT assay (Fig. 1e). In both case, negligible toxicity effect was identified.

It is known that cells internalize extracellular substances via several different pathways. To determine the uptake mechanism of the needle shaped PLGA-PEG fibers, special inhibitors were used to treat cells before co-incubation with the fibers. The uptake ratios of the fibers by the inhibitors treated cells were normalized to those of the control cells and shown in Fig. 2f. Sodium Azide treatment inhibited the cellular uptake amount of the all fibers by $\sim 55 \%$, indicating the presence of an active uptake process [3] since sodium Azide was used as a metabolic inhibitor as it prevents the production of ATP by interfering with glycolytic and oxidative metabolic pathways of cells [4]. Cytochalasin D, a fungal metabolite that could bind to actin filaments and block polymerization and elongation of actin, decreased the cellular uptake of the fibers by $\sim 30 \%$, while Chlorpromazine (inhibitor of clathrin-mediated endocytosis), Genistein (inhibitor of caveolae-mediated endocytosis) or Amiloride (inhibitor of macropinocytosis) failed to alter the cellular uptake amount of the fibers in any significant manner, indicating phagocytosis was the major cellular pathway of the fibers.

It is well known that when particles larger than $500 \mathrm{~nm}$ entered the cells via phagocytosis, they firstly resided in phagosomes $[33,34]$, which usually strictly follow the shape of the internalized object $[8,12,35,36]$. In our case, the Dextran-FITC stained endosomes/lysosomes, which was supposed to be normally spherical shape, took the shape of the internalized fibers (Fig. S4, 

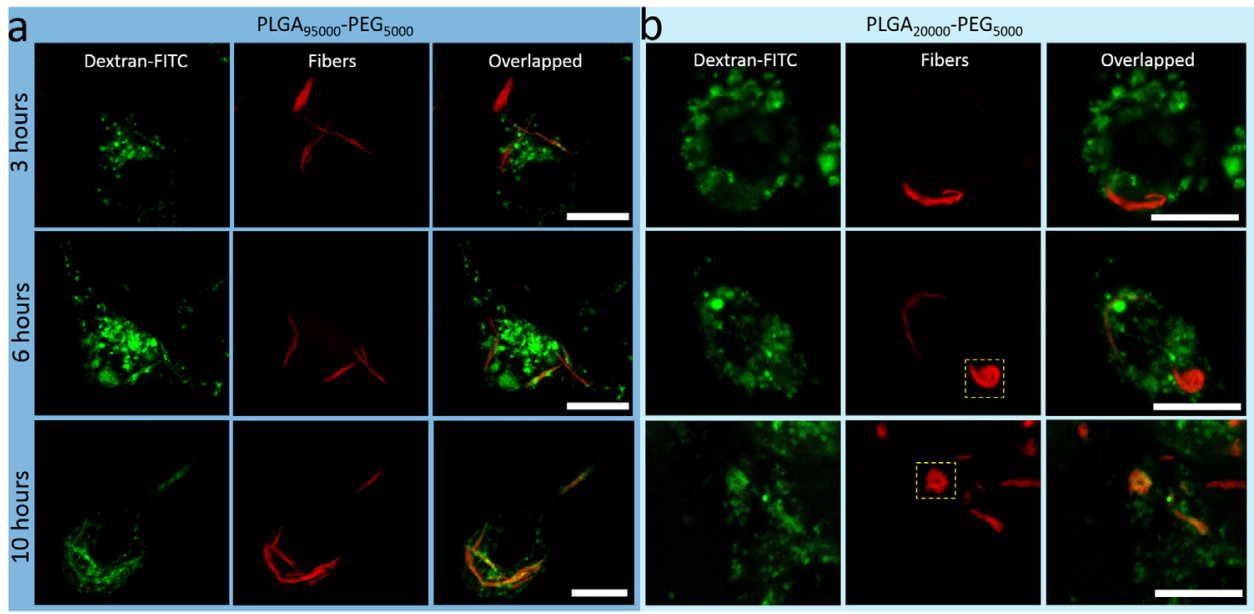

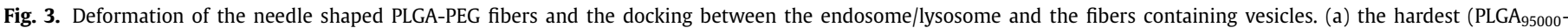

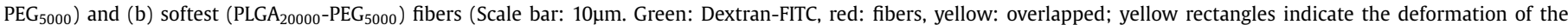
softest fibers).

green channels), indicating these fibers containing vesicles were indeed the late phagocytic vesicles-phagolysosomes. This was consistent with the cellular uptake pathway experiment determined by adding specific inhibitors (Fig. 1f): the fibers entered the cells mainly via phagocytosis.

It is generally accepted that after phagocytosis, docking and fusion between endosome/lysosome and phagosome usually took place [37]. This was indeed observed in the present work when RAW 264.7 cells were fed with different needle shaped PLGAPEG fibers. Taking the hardest PLGA $_{95000}-\mathrm{PEG}_{5000}$ sample as an example, the endosomes/lysosomes (green) were found to dock and fuse with the fibers-containing phagosomes (red) (Fig. 3a, 3 - 6 hrs, \& S5b, 3 - 6 hrs). Intense fusion between the endosomes/lysosomes and the phagosomes can be observed from $\sim 10$ hrs incubation period (Fig. 3a, 10 hrs \& S5b, 10 hrs), while complete fusion was observed at 14 hrs, as indicated by the more and brighter yellow color on the fibers in such a sample (Fig. S5b, 14 hrs). When cells were fed with other fibers, similar phenomena were observed (Fig. 3b, and S5c - f, 3 - 14 hrs). However, ineffective/incomplete fusion was observed in both $\mathrm{PLGA}_{26250}-\mathrm{PEG}_{5000}$ (Fig. S5e, 14 hrs) and PLGA $_{20000}-\mathrm{PEG}_{5000}$ (Fig. S5f, 14 hrs) at the end of $14 \mathrm{hrs}$ ' feeding. The overlap of the fluorescence signals between the fibers and the endosomes/lysosomes reduced from almost $\sim 100 \%$ (in $\mathrm{PLGA}_{95000}-\mathrm{PEG}_{5000}\left(\mathrm{n}=56\right.$ ), $\mathrm{PLGA}_{57500}-\mathrm{PEG}_{5000}$ $(\mathrm{n}=33)$ and $\mathrm{PLGA}_{36700}-\mathrm{PEG}_{5000}(\mathrm{n}=60)$ ) to $\sim 92 \%$ (in $\mathrm{PLGA}_{26250^{-}}$ $\mathrm{PEG}_{5000}(\mathrm{n}=48)$ ) and to $60 \%$ (in $\mathrm{PLGA}_{20000}-\mathrm{PEG}_{5000}(\mathrm{n}=48)$ ), $\mathrm{n}$ represents the number of the counted fibers inside the cells. On the other hand, we also found that the softer fibers $\left(\mathrm{PLGA}_{26250^{-}}\right.$ $\mathrm{PEG}_{5000}$ and $\mathrm{PLGA}_{20000}-\mathrm{PEG}_{5000}$ ) deformed in form of bending and eventually rolling up (Fig. S5e \& f, $6-14 \mathrm{hrs}$ ), which was the most obvious in the $\mathrm{PLGA}_{20000}-\mathrm{PEG}_{5000}$ fibers sample (Fig. 3b).

In order to describe the deformation of the needle shaped PLGA-PEG fibers inside the cells, we use the equivalent length of the internalized fibers as an indicator, that is, length was defined as the farthest distance between two points on individual fibers, and this equivalent length (Fig. 4) is then estimated based on hundreds of fibers measured (Table S3). For samples PLGA ${ }_{95000^{-}}$ $\mathrm{PEG}_{5000}, \mathrm{PLGA}_{57500}-\mathrm{PEG}_{5000}$ and $\mathrm{PLGA}_{36700}-\mathrm{PEG}_{5000}$, the equivalent length of the internalized fibers was almost unchanged when compared to their original length of $\sim 5.5 \mu \mathrm{m}$, indicating no deformation was happened. Little change in the equivalent length is observed in PLGA $_{26200}-\mathrm{PEG}_{5000}$ fibers (second softest) in the first hour after their internalization, followed by a decrease to $\sim 3.6 \mu \mathrm{m}$ at 14 hrs. More significant change in the average length is observed in the softest fibers ( $\mathrm{PLGA}_{20000}-\mathrm{PEG}_{5000}$ ), and a value of $\sim 2.2 \mu \mathrm{m}$ was

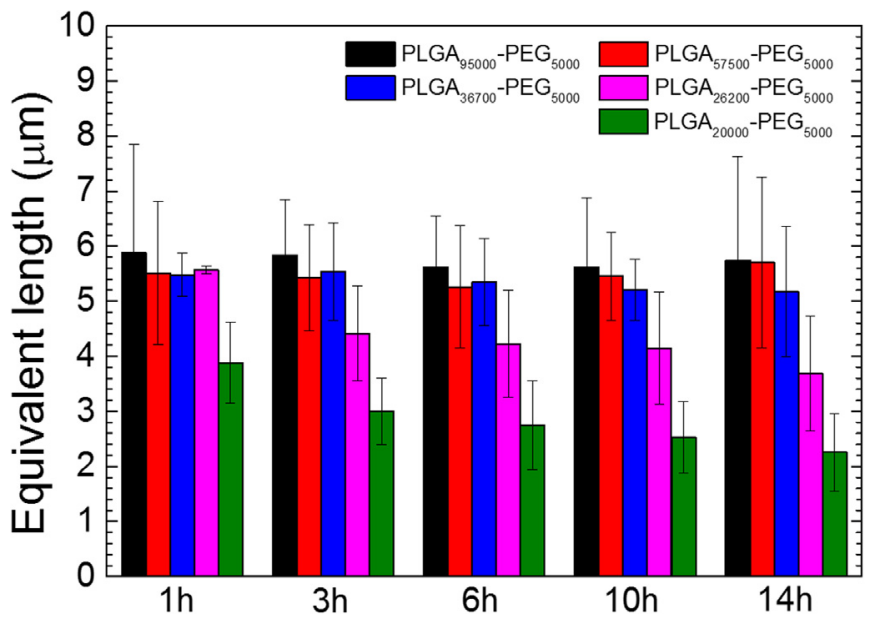

Fig. 4. Equivalent length of needle shaped PLGA-PEG fibers at different time interval, data were measured from CLSM images.

reached at 14 hrs, confirming the deformation of the softer fibers in statistical means.

The deformation observed in the case of softer needle shaped PLGA-PEG fibers ( $\mathrm{PLGA}_{26250}-\mathrm{PEG}_{5000} \& \mathrm{PLGA}_{20000}-\mathrm{PEG}_{5000}$ ) suggested that these fibers were not uniform mechanically. This is consistent with the AFM force mapping obtained on individual fibers (Fig. 1d \& S1).

Evolution of actin on the surface of the fibers containing phagosomes

It was documented in the literature that actin coating on the phagosome impeded the fusion between endosomes/lysosomes and the phagosomes [38]. Consequently, the inefficient fusion between endosome/lysosome and phagosome observed in the case of softer fibers (i.e., $\mathrm{PLGA}_{26250}-\mathrm{PEG}_{5000} \& \mathrm{PLGA}_{20000}-\mathrm{PEG}_{5000}$ ) suggested that the actin coating on the phagosome membrane gathered during the phagocytic process may not leave after the phagocytosis. Therefore, investigating the evolution of actin after phagocytosis became important. Fig. 5a showed the actin absence/presence of the hardest $\left(\mathrm{PLGA}_{95000}-\mathrm{PEG}_{5000}\right)$ and softest $\left(\mathrm{PLGA}_{20000}-\mathrm{PEG}_{5000}\right)$ fibers fed cells at the end of 14 hrs' incubation. Actin coatings on the hardest fibers containing phagosomes almost completely disappeared, as indicated by the little overlap between the fluorescence signals of actin and fibers (Fig. 5b); while 

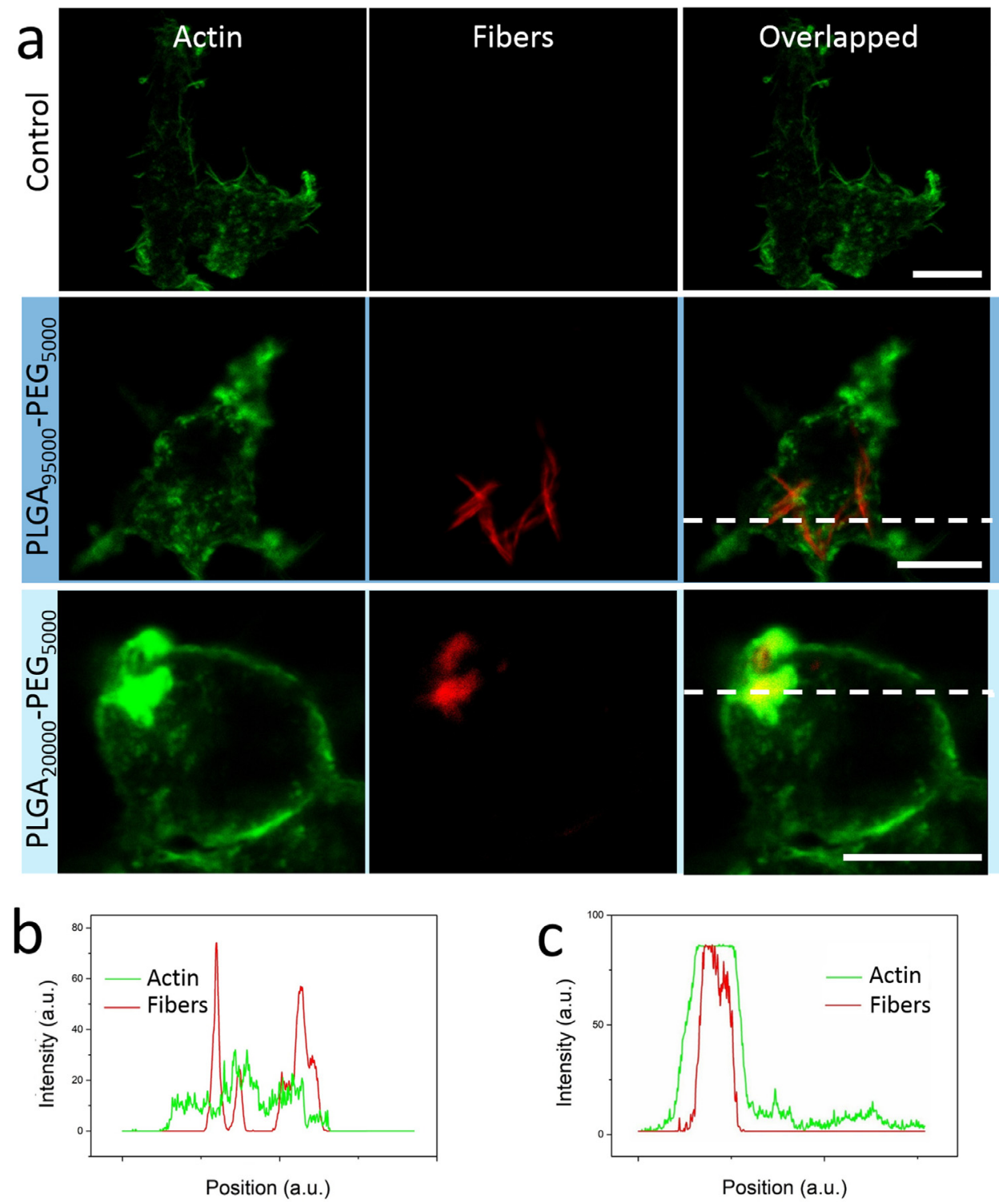

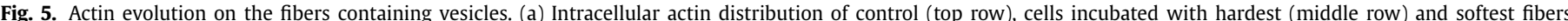

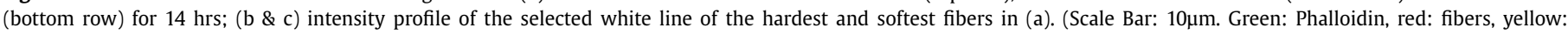
overlapped).

actin coatings on the softest fibers containing phagosomes could always be found easily, as indicated by the largely overlapped fluorescence signals of actin and fibers (Fig. 5c). This result suggested that the actin coating remained on the softer fibers containing phagosomes, but not those with harder ones.

Similar trends can be found in others samples. As shown in Figure S6, actin activity became the most active in the first 3 hrs of phagocytosis of all fiber samples, when actin covering on the fiber-containing phagosomes were easy to be found. Shortly after that, actin can still be found covering on some of the fibers containing phagosomes, then the number of actin coated phagosomes reduced and almost completely disappeared at 14 hrs for cells fed with harder fibers $\left(\mathrm{PLGA}_{95000}-\mathrm{PEG}_{5000}, \mathrm{PLGA}_{57500}-\mathrm{PEG}_{5000}\right.$ and $\mathrm{PLGA}_{36700}-\mathrm{PEG}_{5000}$ ), as indicated by the little overlap in the fluorescence signals of actin and fibers (Fig. 5a, S6b, c \& d, 14 hrs). When cells were fed with softer fibers (i.e., $\mathrm{PLGA}_{26250}-\mathrm{PEG}_{5000}$ \& PLGA $_{20000}-\mathrm{PEG}_{5000}$ ), similar phenomena can be observed in the first 3 hrs (Fig. S6e \& f, 1 - 3 hrs). However, actin coated phagosomes can always be found (Fig. 5a, S6e \& f, after 3 hrs) after long feeding duration (up to $14 \mathrm{hrs}$ ), as indicated by the overlapped fluorescence signals of actin and fibers. In the case of the softest fibers $\left(\mathrm{PLGA}_{20000}-\mathrm{PEG}_{5000}\right)$, some actin coated vesicles even remained up to $24 \mathrm{hrs}$ (Fig. S6f, 18 \& $24 \mathrm{hrs}$ ).

The intracellular trafficking/deformation of the needle shaped PLGA-PEG fibers and the evolution of actin on the surface of the fibers-containing phagosomes suggested a possible correlation between the actin and the deformation of the softer fibers. The judgement of whether the actin coatings caused the softer fibers' deformation was verified by the following experiments. All the fibers were fed to cells at the very beginning, 3 hrs latter the polymerization of actin was knocked down by adding Cytochalasin D to the same medium and another 11 hrs incubation was followed. After that, all the fibers were found to keep their equivalent length the same as they were at $3 \mathrm{hrs}$ (Table S4 and Fig. S7), confirming the fibers' deformation was caused by actin.

We also tried to exclude the possibility that actin preferred to cover vesicles with low aspect ratio. In this set of experiments, two extreme cases were chosen: the precursors of the hardest $\left(\mathrm{PLGA}_{95000}-\mathrm{PEG}_{5000}\right)$ and the softest $\left(\mathrm{PLGA}_{20000}-\mathrm{PEG}_{5000}\right)$ fibers - their spherical counter parts, with diameter $\sim 450 \mathrm{~nm}$ of the 
same volume, were fed to cells, after that the actin evolution was recorded by CLSM. As shown in Figure S8, during the first 3 hrs' incubation, most of the spheres containing vesicles were covered with actin, and no obvious deformation of the spheres could be observed. This was consistent with the observation made on the needle shaped PLGA-PEG fibers with much higher aspect ratio 25. After that, the actin coating started to dissipate in both samples as time elapsing and was no longer detectable at 14 hrs' incubation. Such actin behavior was very similar to that observed in the harder fibers' case - actin covered the fibers containing vesicles at the very beginning after feeding, and then tried to deform the fibers, if the fibers could not be deformed, actin started to dissipate. These results indicated that the actin dissipation was independent of the particle shape; but relied on whether the cargo inside the vesicle can deform or not.

\section{Discussion}

Deformation of particles had been reported to take place at the early stage of phagocytosis [30,39,40], e.g., upon anchoring to the cell membrane, soft particles (with Young's modulus $760 \mathrm{MPa}$ ) were found to deform more in response to phagocytic force exerted by the actin driven membrane protrusion than the hard ones ( 1.2 GPa) [30]. The deformation of the particles was described by the changes in its radius of curvature at the contact point with the cell membrane. If the deformation is significant enough to cause shape changes, i.e., from spherical shape to rod-like shape, reduced phagocytosis rate was further induced, as understood by the energy increase required by plasma membrane wrapping during phagocytosis in theoretical model $[12,41]$. Nevertheless, once the particles were internalized, it was generally accepted that the shape of the phagosome took after that of the particles, and would not change afterwards[8,12,35,36]. A surprising discovery in the present work is the observation of particle deformation (i.e., bending and rolling up) at $6 \mathrm{hrs}$ after their internalization into the intracellular environment in the case of the two softer samples $\left(\mathrm{PLGA}_{26250}-\mathrm{PEG}_{5000}\right.$ and $\left.\mathrm{PLGA}_{20000}-\mathrm{PEG}_{5000}\right)$.

The experimental observation of (1) constantly observed actin coatings on the vesicles containing two softer fibers ( $\mathrm{PLGA}_{26250^{-}}$ $\mathrm{PEG}_{5000}$ and $\mathrm{PLGA}_{20000}-\mathrm{PEG}_{5000}$ ), but not on those containing the harder ones $\left(\mathrm{PLGA}_{95000}-\mathrm{PEG}_{5000}, \mathrm{PLGA}_{575000}-\mathrm{PEG}_{5000}\right.$ and $\mathrm{PLGA}_{36700}-\mathrm{PEG}_{5000}$ ); (2) long lasting actin presence on the vesicles containing softer fibers (14 hrs for PLGA $26250-\mathrm{PEG}_{5000}$ and up to 24 hrs for $\mathrm{PLGA}_{20000}-\mathrm{PEG}_{5000}$ ) and the quick actin depletion (within 6 hrs) followed by complete fusion of fibers containing phagosome and endosome/lysosome in the case of harder samples (PLGA ${ }_{95000^{-}}$ $\mathrm{PEG}_{5000}, \mathrm{PLGA}_{575000}-\mathrm{PEG}_{5000}$ and $\mathrm{PLGA}_{36700}-\mathrm{PEG}_{5000}$ ); and (3) little deformation of the fibers when actin polymerization was inhibited, strongly suggest the correlation between the presence of actin coating and the driving force for the fibers' deformation. In fact, it had been found that actin coating deposited on the surface of lipid vesicles, and exerted compressive force to its interior in simulated solution of the intracellular environment. [42,43].

Few actin related mechanical models can be found in the literature, with either experimental $[40,43]$ or theoretical origins $[44,45]$. Nevertheless, all of these models were built to explain the motion of the vesicles driven by the asymmetrical actin polymerization or breaking down of the actin coating. As the observation in the present experiment related to bending and curling of the fibers, we used a simple strut bucking model to estimate the compressive force and pressure of the actin based on the apparent Young's modulus of the fibers measured by AFM (Figure S9). Considering a strut with similar geometry to the fibers and apparent Young's modulus ranging from 5 to $140 \mathrm{kPa}$ to simulate the softest fiber, we calculated the minimum compressive force for initialing the bending was $4.54 \mathrm{pN}$, and the corresponding pressure of the actin were $212.6 \mathrm{~Pa}$, which located within the compressive force range in the literature reports of the F-actin network, that is, in the range of $-4 \times 10^{-3}$ to $5 \times 10^{3} \mathrm{~Pa}[43,46]$.

\section{Conclusion}

We synthesized needle shaped PLGA-PEG fibers with different apparent Young's modulus and investigated how their deformation affect the physiological response of macrophages. We found that the softest fibers inside the phagosome were deformed by the surrounding actin coatings, and a reluctance of actin depletion of the phagosomes containing the softest fibers. The interplay between fibers' deformation and actin response disclosed the important role of material mechanical property in affecting the physiology of cells. The different cellular fate of soft fibers vs. hard fibers (long lasting phagosome environment vs. lysosome environment) suggested mechanical engineering serve as effective means to tailor nanoparticle carriers design for special delivery purpose. On the other hand, the deformation of the soft fiber driven by actin might potentially provide an alternative approach for in situ measuring the mechanical properties of biomaterials in live systems.

\section{Declaration of Competing Interest}

The authors declare that they have no known competing financial interests or personal relationships that could have appeared to influence the work reported in this paper.

\section{Acknowledgement}

This work is supported in part by the Hong Kong Collaborative Research Fund (CRF) Scheme (Ref. C4026-17W) and Theme-based Research Scheme (Ref. T13-402/17-N).

\section{Supplementary materials}

Supplementary material associated with this article can be found, in the online version, at doi:10.1016/j.actbio.2020.05.029.

\section{References}

[1] L. Shang, K. Nienhaus, G.U. Nienhaus, Engineered nanoparticles interacting with cells: size matters, J Nanobiotechnol 12 (2014) b26.

[2] L. Shang, K. Nienhaus, X. Jiang, L. Yang, K. Landfester, V. Mailänder, T. Simmet, G.U. Nienhaus, Nanoparticle interactions with live cells: quantitative fluorescence microscopy of nanoparticle size effects, Beilstein J. Nanotechnol. 5 (2014) 2388-2397

[3] W. Jiang, B.Y.S. Kim, J.T. Rutka, W.C.W. Chan, Nanoparticle-mediated cellular response is size-dependent, Nat. Nanotechnol. 3 (2008) 145-150.

[4] R.R. Arvizo, O.R. Miranda, M.A. Thompson, C.M. Pabelick, R. Bhattacharya, J.D. Robertson, V.M. Rotello, Y.S. Prakash, P. Mukherjee, Effect of Nanoparticle Surface Charge at the Plasma Membrane and Beyond, Nano Lett 10 (2010) 2543-2548.

[5] A. Verma, F. Stellacci, Effect of Surface Properties on Nanoparticle-Cell Interactions, Small 6 (2010) 12-21.

[6] E.L. da Rocha, G.F. Caramori, C.R. Rambo, Nanoparticle translocation through a lipid bilayer tuned by surface chemistry, Phys Chem Chem Phys 15 (2013) 2282-2290.

[7] Y. Zhang, D. Xu, W. Li, J. Yu, Y. Chen, Effect of Size, Shape, and Surface Modification on Cytotoxicity of Gold Nanoparticles to Human HEp-2 and Canine MDCK Cells, J. Nanomater. 2012 (2012) 1-7.

[8] J.A. Champion, S. Mitragotri, Role of target geometry in phagocytosis, Proc Natl. Acad. Sci. U. S. A. 103 (2006) 4930-4934.

[9] S. Kumar, A.C. Anselmo, A. Banerjee, M. Zakrewsky, S. Mitragotri, Shape and size-dependent immune response to antigen-carrying nanoparticles, J. Controlled Release. 220 (2015) 141-148.

[10] D.H. Jo, J.H. Kim, T.G. Lee, J.H. Kim, Size, surface charge, and shape determine therapeutic effects of nanoparticles on brain and retinal diseases, Nanomedicine Nanotechnol. Biol. Med. 11 (2015) 1603-1611.

[11] A. Albanese, P.S. Tang, W.C.W. Chan, The Effect of Nanoparticle Size, Shape, and Surface Chemistry on Biological Systems, Annu. Rev. Biomed. Eng. 14 (2012) $1-16$.

[12] S. Dasgupta, T. Auth, G. Gompper, Shape and Orientation Matter for the Cellular Uptake of Nonspherical Particles, Nano Lett 14 (2014) 687-693. 
[13] P.A. Janmey, C.A. McCulloch, Cell Mechanics, Integrating Cell Responses to Mechanical Stimuli, Annu. Rev. Biomed. Eng. 9 (2007) 1-34.

[14] V. Mailänder, K. Landfester, Interaction of Nanoparticles with Cells, Biomacromolecules 10 (2009) 2379-2400.

[15] F. Zhao, Y. Zhao, Y. Liu, X. Chang, C. Chen, Y. Zhao, Cellular uptake, intracellular trafficking, and cytotoxicity of nanomaterials, Small 7 (2011) 13221337.

[16] R.A. Petros, J.M. DeSimone, Strategies in the design of nanoparticles for therapeutic applications, Nat. Rev. Drug Discov. 9 (2010) 615-627.

[17] A.E. Nel, L. Mädler, D. Velegol, T. Xia, E.M.V. Hoek, P. Somasundaran, F. Klaessig, V. Castranova, M. Thompson, Understanding biophysicochemical interactions at the nano-bio interface, Nat. Mater. 8 (2009) 543-557.

[18] J.A. Champion, Y.K. Katare, S. Mitragotri, Making polymeric micro- and nanoparticles of complex shapes, Proc. Natl. Acad. Sci. 104 (2007) 11901-11904.

[19] R.J. Mondschein, A. Kanitkar, C.B. Williams, S.S. Verbridge, T.E. Long, Polymer structure-property requirements for stereolithographic 3D printing of soft tissue engineering scaffolds, Biomaterials 140 (2017) 170-188.

[20] C. Kung, A possible unifying principle for mechanosensation, Nature 436 (2005) 647-654

[21] B. Ladoux, R.-M. Mège, Mechanobiology of collective cell behaviours, Nat. Rev. Mol. Cell Biol. 18 (2017) 743-757.

[22] W. Liu, X. Zhou, Z. Mao, D. Yu, B. Wang, C. Gao, Uptake of hydrogel particles with different stiffness and its influence on HepG2 cell functions, Soft Matter 8 (2012) 9235.

[23] M.F. Bédard, A. Munoz-Javier, R. Mueller, P. del Pino, A. Fery, W.J. Parak, A.G. Skirtach, G.B. Sukhorukov, On the mechanical stability of polymeric microcontainers functionalized with nanoparticles, Soft Matter 5 (2009) 148-155.

[24] H. Sun, E.H.H. Wong, Y. Yan, J. Cui, Q. Dai, J. Guo, G.G. Qiao, F. Caruso, The role of capsule stiffness on cellular processing, Chem. Sci. 6 (2015) 3505-3514.

[25] A. Garapaty, J.A. Champion, Tunable particles alter macrophage uptake based on combinatorial effects of physical properties: Garapaty and Champion, Bioeng. Transl. Med. 2 (2017) 92-101.

[26] X. Chen, J. Cui, H. Sun, M. Müllner, Y. Yan, K.F. Noi, Y. Ping, F. Caruso, Analysing intracellular deformation of polymer capsules using structured illumination microscopy, Nanoscale 8 (2016) 11924-11931.

[27] A.C. Anselmo, M. Zhang, S. Kumar, D.R. Vogus, S. Menegatti, M.E. Helgeson, S. Mitragotri, Elasticity of Nanoparticles Influences Their Blood Circulation, Phagocytosis, Endocytosis, and Targeting, ACS Nano 9 (2015) 3169-3177.

[28] L. Zhang, Z. Cao, Y. Li, J.-R. Ella-Menye, T. Bai, S. Jiang, Softer Zwitterionic Nanogels for Longer Circulation and Lower Splenic Accumulation, ACS Nano 6 (2012) 6681-6686.

[29] X. Yi, X. Shi, H. Gao, Cellular Uptake of Elastic Nanoparticles, Phys. Rev. Lett. (2011) 107.
[30] J. Sun, L. Zhang, J. Wang, Q. Feng, D. Liu, Q. Yin, D. Xu, Y. Wei, B. Ding, X. Shi, X. Jiang, Tunable Rigidity of (Polymeric Core)-(Lipid Shell) Nanoparticles for Regulated Cellular Uptake, Adv. Mater. 27 (2015) 1402-1407.

[31] Y. Xia, J. Wu, W. Wei, Y. Du, T. Wan, X. Ma, W. An, A. Guo, C. Miao, H. Yue, S. Li, X. Cao, Z. Su, G. Ma, Exploiting the pliability and lateral mobility of Pickering emulsion for enhanced vaccination, Nat. Mater. 17 (2018) 187-194.

[32] P. Xu, E. Gullotti, L. Tong, C.B. Highley, D.R. Errabelli, T. Hasan, J.-X. Cheng, D.S. Kohane, Y. Yeo, Intracellular Drug Delivery by Poly(lactic- co -glycolic acid) Nanoparticles, Revisited, Mol. Pharm. 6 (2009) 190-201.

[33] J. Fang, J.A. Brzostowski, S. Ou, N. Isik, V. Nair, T. Jin, A vesicle surface tyrosine kinase regulates phagosome maturation, J. Cell Biol. 178 (2007) 411-423.

[34] B. Alberts, Molecular Biology of the Cell, Courier Corporation (1989).

[35] N. Daum, C. Tscheka, A. Neumeyer, M. Schneider, Novel approaches for drug delivery systems in nanomedicine: effects of particle design and shape, Wiley Interdiscip. Rev. Nanomed. Nanobiotechnol. 4 (2012) 52-65.

[36] J. Zhao, M.H. Stenzel, Entry of nanoparticles into cells: the importance of nanoparticle properties, Polym. Chem. 9 (2018) 259-272.

[37] W. Stockinger, S.C. Zhang, V. Trivedi, L.A. Jarzylo, E.C. Shieh, W.S. Lane, A.B. Castoreno, A. Nohturfft, Differential requirements for actin polymerization, calmodulin, and $\mathrm{Ca} 2+$ define distinct stages of lysosome/phagosome targeting, Mol. Biol. Cell. 17 (2006) 1697-1710.

[38] D. Liebl, G. Griffiths, Transient assembly of F-actin by phagosomes delays phagosome fusion with lysosomes in cargo-overloaded macrophages, J, Cell Sci 122 (2009) 2935-2945.

[39] K.A. Beningo, Y. Wang, Fc-receptor-mediated phagocytosis is regulated by mechanical properties of the target, J. Cell Sci. 115 (2002) 849-856.

[40] H. Gao, Probing mechanical principles of cell-nanomaterial interactions, J. Mech. Phys. Solids. 62 (2014) 312-339.

[41] A.H. Bahrami, M. Raatz, J. Agudo-Canalejo, R. Michel, E.M. Curtis, C.K. Hall, M. Gradzielski, R. Lipowsky, T.R. Weikl, Wrapping of nanoparticles by membranes, Adv. Colloid Interface Sci. 208 (2014) 214-224.

[42] P. Miklavc, K. Ehinger, A. Sultan, T. Felder, P. Paul, K.-E. Gottschalk, M. Frick, Actin depolymerisation and crosslinking join forces with myosin II to contract actin coats on fused secretory vesicles, J. Cell Sci. 128 (2015) 1193-1203.

[43] P.A. Giardini, D.A. Fletcher, J.A. Theriot, Compression forces generated by actin comet tails on lipid vesicles, Proc. Natl. Acad. Sci. 100 (2003) 6493-6498.

[44] M.J. Dayel, O. Akin, M. Landeryou, V. Risca, A. Mogilner, R.D. Mullins, In Silico Reconstitution of Actin-Based Symmetry Breaking and Motility, PLoS Biol 7 (2009) e1000201.

[45] J. Zhu, A. Mogilner, Mesoscopic Model of Actin-Based Propulsion, PLoS Comput. Biol. 8 (2012) e1002764.

[46] A. Upadhyaya, J.R. Chabot, A. Andreeva, A. Samadani, A. van Oudenaarden, Probing polymerization forces by using actin-propelled lipid vesicles, Proc. Natl. Acad. Sci. 100 (2003) 4521-4526. 